\title{
The impact of Aurora kinase A genetic polymorphisms on cervical cancer progression and clinicopathologic characteristics
}

\author{
Pei-Ju Wu1,2,3\#, Chun-Hao Wang4\#, Ming-Hong Hsieh²,5, Chung-Yuan Lee6,7, Po-Hui Wang 1,2,3, Chiao-Wen \\ Lin $^{8}$, Shun-Fa Yang1, ${ }^{\bowtie}$ and Maw-Sheng Lee ${ }^{1,2,3 凶}$ \\ 1. Institute of Medicine, Chung Shan Medical University, Taichung, Taiwan. \\ 2. School of Medicine, Chung Shan Medical University, Taichung, Taiwan. \\ 3. Department of Obstetrics and Gynecology, Chung Shan Medical University Hospital, Taichung, Taiwan. \\ 4. Department of Medicine, National Taiwan University, Taipei, Taiwan. \\ 5. Department of Psychiatry, Chung Shan Medical University Hospital, Taichung, Taiwan. \\ Department of Obstetrics and Gynecology, Chiayi Chang Gung Memorial Hospital, Chiayi, Taiwan. \\ . Department of Nursing, Chang Gung University of Science and Technology, Chiayi Campus, Chiayi, Taiwan \\ Institute of Oral Sciences, Chung Shan Medical University, Taichung, Taiwan. \\ . Department of Medical Research, Chung Shan Medical University Hospital, Taichung, Taiwan. \\ \#Equal contribution as first authors. \\ $\triangle$ Corresponding authors: Shun-Fa Yang, PhD or Maw-Sheng Lee, MD, PhD. Institute of Medicine, Chung Shan Medical University, 110, Section 1, Chien-Kuo North Road, \\ Taichung, 40201, Taiwan. Tel.: 886-4-24739595 ext. 21721; Fax: 884-4-24738493. E-mail: ysf@csmu.edu.tw (Shun-Fa Yang); msleephd@gmail.com (Maw-Sheng Lee). \\ (C) The author(s). This is an open access article distributed under the terms of the Creative Commons Attribution License (https://creativecommons.org/licenses/by/4.0/). \\ See http://ivyspring.com/terms for full terms and conditions.
}

Received: 2021.01.22; Accepted: 2021.04.04; Published: 2021.04.22

\begin{abstract}
The aims of this study were to explore the involvement of Aurora kinase A (AURKA) gene single nucleotide polymorphisms (SNPs) in uterine cervical cancer that has not yet been investigated. One hundred and six patients with cervical invasive cancer and 94 patients with precancerous lesions, and 302 Taiwanese female individuals were included. AURKA SNPs rs2273535, rs6024836, rs2064863 and rs1047972 were analyzed for genotypic distributions using real-time polymerase chain reaction. There were no statistically significant differences in the genetic frequencies of AURKA SNPs among patients with invasive cancer and those with precancerous lesions of uterine cervix and control women. There were no associations among AURKA SNPs and clinicopathologcal variables and recurrence and survival events. However, in a multivariate analysis, cervical cancer patients with adenocarcinoma (HR: 3.18, 95\% Cl: 1.23-8.23; $\mathrm{p}=0.017)$ and larger tumor (HR: $5.61,95 \%$ $\mathrm{Cl}$ : 2.10-14.95; $p=0.001$ ) had poorer recurrence-free survival. In conclusion, tumor size and pelvic lymph node status rather than AURKA SNPs were the most obvious independent parameter that could significantly predict 5 years survival rate in Taiwanese women with cervical cancer.
\end{abstract}

Key words: Aurora kinase A, single nucleotide polymorphisms, uterine cervical cancer, pelvic lymph node metastasis

\section{Introduction}

Uterine cervical cancer still remains an important public health issue, leading to a considerable morbidity and mortality rate $[1,2]$. It is the eighth most common malignant tumor among women and the second most common gynecological malignancy in Taiwan. According to the Taiwan Annual Cancer Registry Report it revealed the age-standardized incidence rate of cervical cancer in 2014 to be 8.50 per 100000 women from the Health Promotion Administration of the Ministry of Health and Welfare. The age-standardized mortality rate of cervical cancer was 3.39 per 100000 women in 2014, which was the seventh leading cause of cancer deaths among women in Taiwan.0

A continuous and multi-step process is required for the formation of invasive cancer of uterine cervix. The multi-step process consists of neoplastic transformation from cervical intraepithelial neoplasia (CIN), known as precancerous lesions, to invasive cancer, which was regarded as the end of CIN progression [3,4]. When mitoses and immature cells occupy in the lower one-third of cervical epithelium, 
it is referred to CIN 1 histologically (also regarded as low-grade CIN or dysplasia, or mild dysplasia) or low-grade squamous cell intraepithelial lesions (LSIL), for cytological counterpart. When mitoses and immature cells progress in the middle and upper third epithelium, CIN 2 (moderate dysplasia) and CIN 3 (severe dysplasia and carcinoma in situ when whole epithelium is involved) are denominated histologically, respectively. They are collectively regarded as high-grade CIN or high-grade dysplasia and considered as precancerous lesions. The cytological counterpart is called high-grade squamous cell intraepithelial lesions (HSIL) [5].

Aurora kinase A (AURKA) is located at chromosome 20q13.2 and belongs to Aurora kinases, a family, which consists of Aurora kinase A, B (AURKB) and $C$ (AURKC) [6, 7]. It functions as a check-point-associated kinase in the cell cycle, and is linked to the regulation of transition from G2 to M phase in cell division [7-9]. In addition to focusing on cell division, it has been reported to also regulate the self-renewal and reprogramming of stem cells $[10,11]$. However, its overexpression inhibits BRAC1 and BRCA2, which may repair DNA double strand breaks $[12,13]$. AURKA overexpression was also found to be significantly related to high-grade and high-stage hepatocellular carcinoma tumors [14]. In addition, AUKRA amplification has been found in a variety of cancers, such as breast and ovarian cancers $[15,16]$.

If a single nucleotide difference in the shared sequence of a gene in a pair of chromosomes exceeds $1 \%$ of certain populations, it is defined as a single nucleotide polymorphism (SNP)[17-20]. When SNPs exhibit an influence on the promoter area, exon and 3 '-untranslated region of a gene, it may affect the gene expression, thereby promote the occurrence of disease and cancer [17, 21-23]. Numerous SNPs in AUKRA genes, including rs2273535, rs6024836, rs2064863 and rs1047972, have been identified as being associated with cancer progression and cancer susceptibility [24-27]. To the best of our knowledge, no study investigates the relationships among AURKA genetic polymorphisms and cervical tumorigenesis in Taiwanese female individuals, as well as the clinicopathological characteristics and patient survival of cervical cancer. Therefore, we conducted this study to delineate the involvement of AURKA in the cervical carcinogenesis and clinical variables and patient survival of cervical cancer.

\section{Materials and methods}

\section{Female individuals}

The Department of Obstetrics and Gynecology of the Affiliated Hospital of Chung Shan Medical
University in Taiwan enrolled 106 patients with invasive cancer and 94 patients with precancerous lesions of uterine cervix. Meanwhile, 302 female individuals without cervical lesions were included as a control group. All subjects lived in central Taiwan, where is our hospital. Therefore, the study population was recruited from this area. In our hospital, the incidence of cervical cancer is similar to other regions of Taiwan. Patients with cervical precancerous lesions or invasive cancer had undergone standard treatment protocols at the Department of Obstetrics and Gynecology in Chung Shan Medical University Hospital between January 2000 and December 2014. The clinical staging of cervical cancer patients was based on the 2009 International Federation of Obstetrics and Gynecology system. The official pathology report confirmed the diagnosis of cervical invasive carcinoma and high-grade CIN. Neither SIL lesions nor cancers cells were found in the cervical smear reports of control women. These smears examinations were done in the outpatient clinic of the Affiliated Hospital of Chung Shan Medical University, and the cytological diagnosis was further verified by colposcopy to meet the results of the smear examination. The study was conducted under the approval of the Institutional Review Board of the Chung Shan Medical University Hospital (CSMUH number: CS18208), and everyone's informed consent was obtained.

\section{Deoxyribonucleic acid (DNA) extraction from blood samples of all participants and selection of AURKA SNPs}

Laboratory staff used venipuncture techniques to draw blood samples from all participants. The samples were collected in a Vacutainer tubes mixed with ethylenediaminetetraacetic acid. They were stored at $4{ }^{\circ} \mathrm{C}$ immediately. DNA was extracted using the QIAamp DNA Blood Mini Kit according to the manufacturer's instructions [28, 29]. The recruited DNA was therefore dissolved in $\mathrm{pH} 7.8$ TE buffer. Then, it was quantified by the measurement of OD260. The OD260/OD280 ratio was checked and the range of 1.8-2.0 met our standards and defined as pure to prevent it from cross-reacting with the current homologous RNA in the samples. The final product were then stored at $-20^{\circ} \mathrm{C}$ and used as a template for the polymerase chain reaction (PCR).

Based on the data of the international HapMap project and previous publication by Mesic et al., four AURKA SNPs were selected [30]. AURKA genetic variants rs2273535, rs6024836, rs2064863 and rs1047972 were examined through ABI StepOne Real-Time PCR System (Applied Biosystems, Foster 
City, CA, USA), and determined with SDS vers. 3.0 software, as our previous publication [26].

\section{Statistical analysis}

Analysis of variance (ANOVA) was used to compare the age distribution of the female individuals studied, and Scheffe test was applied for post hoc analysis. The Hardy-Weinberg equilibrium was performed to examine the genotypic frequencies of AURKA rs2273535, rs6024836, rs2064863 and rs1047972 in normal controls [degree of freedom (d.f.) $=2]$.

In order to define the relationships between AURKA SNPs and cervical neoplasias, chi-square or Fisher's exact tests was done. The adjusted odds ratios (AORs) with their 95\% confidence intervals (CIs) were calculated to check the relationships among genotypic distributions of AURKA SNPs and the incidence of cervical neoplasias (including precancerous lesions and invasive cancer) through the logistic and multinomial logistic regression models for controlling age of the subjects.

Chi-square or Fisher's exact tests were used to define the relationships among AURKA SNPs and clinicopathological parameters as well as recurrence and mortality events of cervical cancer. Kaplan-Meier curve model (univariate analysis over time) was used to determine the significance of AURKA SNPs and clinicopathological parameters in the prognoses of patients, which were statistically related to with recurrence-free and overall survival of cervical cancer patients. The log-rank test was tested to examine the differences among them. In the multivariate analysis of recurrence-free and overall survival time, a Cox proportional hazard model of a forward stepwise method was used to examine the influences of AURKA genetic variants and a variety of clinicopathological factors on recurrence-free and overall survival. Thus, the hazard ratios (HRs) were calculated. The statistical analysis of SPSS, version 18.0 and WinPepi Software, version 10.0 was done for statistical significance. $p<0.05$ was regarded as statistically significant difference.

\section{Results}

It indicated that there was significant difference in the age distribution between patients with cervical neoplasias and normal control women $(50.1 \pm 14.0$ vs. $43.8 \pm 10.0, p<0.001)$. A post hoc analysis based on the Scheffe test of the ANOVA found that the age distribution between patients with cervical invasive cancer and those with precancerous lesions was statistically different $(55.6 \pm 12.8$ vs. $43.9 \pm 12.7$, $p<0.001$ ), as well as statistically different between patients with cervical cancer and control women (55.6 \pm 12.8 vs. $43.8 \pm 10.0, p<0.001$ ) but not statistically different between those with precancerous lesions and control women $(43.9 \pm 12.7$ vs. $43.8 \pm 10.0, p=$ 0.999).

The minor allele frequencies of AURKA genetic variants rs2273535, rs6024836, rs2064863 and rs1047972 in control female individuals were all $\geq 5 \%$. Genotypic frequency of AURKA genetic variant rs2273535 conformed to the Hardy-Weinberg equilibrium [ $\chi^{2}$ value, $0.114, p=0.945$; d.f. $=2$ ] in control females. The frequencies of rs6024836, rs2064863 and rs1047972 were also satisfied the Hardy-Weinberg equilibrium ( $\chi^{2}$ value, 3.976, $p=0.137 ; \chi^{2}$ value, 0.367, $p=0.833$ and $\chi^{2}$ value, $0.093, p=0.955$; respectively).

Table 1 lists genetic polymorphism frequencies of AURKA gene in Taiwanese women with cervical neoplasias and normal controls. There were no significantly different in the distributions of AURKA SNPs rs2273535, rs6024836, rs2064863 and rs1047972 between patients with cervical neoplasias and normal control women. After controlling for age, there were still no different distributions between patients with cervical neoplasias and normal controls. After categorizing cervical neoplasias into precancerous lesions and invasive cancer subgroups, Table 2 reveals the genotypic frequencies of AURKA gene in patients with cervical invasive cancer and precancerous lesions as well as normal controls. Even after adjusting for age, there were no significant differences in the distributions of AURKA SNPs rs2273535, rs6024836, rs2064863 and rs1047972 among patients with cervical invasive cancer and precancerous lesions as well as normal control women.

When AURKA SNPs were related to the clinicopathological parameters, no statistical significances were found among them (Table 3). We further investigate the associations of AURKA SNPs with recurrence and survival events of cervical cancer patients. Neither AURKA rs6024836 nor other AURKA SNPs are associated with recurrence and mortality events of cervical cancer patients (Table 4). But, cervical cancer patients accompanied by adenocarcinoma, deeper stromal invasion, larger tumor diameter, positive vagina invasion and positive pelvic lymph node metastasis had a higher risk of recurrence event. Furthermore, patients with clinical stage $\geq$ stage II, deeper stromal invasion, larger tumor diameter, positive parametrium and vagina invasion as well as positive pelvic lymph nodes metastasis exhibited a higher risk of mortality event (Table 4). However, it did not reveal different distributions of rs2273535, rs6024836, rs2064863 and rs1047972 among patients with different clinical stage, different pathological type, stromal invasion depth, tumor size, parametrium and vagina invasive conditions and 
pelvic lymph node metastasis status of cervical cancer patients.

In the Kaplan-Meier curve univariate analysis model, AURKA rs6024836 was not related to recurrence-free survival and overall survival of cervical cancer patients (Table 5). Other AURKA SNPs also did not play any statistical significance in the prognoses of these patients. Cervical cancer patients, who exhibited clinical stage $\geq$ stage II, adenocarcinoma, deeper stromal invasion, larger tumor diameter, positive vagina invasion and positive pelvic lymph node metastasis, had poorer recurrence-free survival. Cervical cancer patients with $\geq$ stage II clinical stage, deeper stromal invasion, larger tumor diameter, positive parametrium and vagina invasions and positive pelvic lymph node metastasis had poorer overall survival (Table 5).

Table 1. Distributions of genetic polymorphism of aurora kinase A gene in women with uterine cervical neoplasias and normal controls in Taiwan

\begin{tabular}{|c|c|c|c|c|c|c|}
\hline Genetic polymorphisms & Normal controls $(n=302)$ & Cervical neoplasiasa $(n=200)$ & ORs (95\% CIs) & $p$ values & AORs $(95 \% \text { CIs })^{b}$ & Adjusted $p$ values ${ }^{b}$ \\
\hline rs2273535 & & & & 0.730 & & 0.936 \\
\hline TTc & 138 & 96 & 1.00 & & 1.00 & \\
\hline TA & 134 & 88 & $0.94(0.65-1.37)$ & 0.763 & $0.97(0.66-1.43)$ & 0.869 \\
\hline AA & 30 & 16 & $0.77(0.40-1.48)$ & 0.430 & $0.88(0.45-1.74)$ & 0.721 \\
\hline TTc & 138 & 96 & 1.00 & 0.612 & 1.00 & 0.800 \\
\hline $\mathrm{TA} / \mathrm{AA}$ & 164 & 104 & $0.91(0.64-1.30)$ & & $0.95(0.66-1.38)$ & \\
\hline $\mathrm{TT} / \mathrm{TA}^{\mathrm{c}}$ & 272 & 184 & 1.00 & 0.463 & 1.00 & 0.746 \\
\hline AA & 30 & 16 & $0.79(0.42-1.49)$ & & $0.90(0.47-1.72)$ & \\
\hline rs6024836 & & & & 0.855 & & 0.543 \\
\hline $\mathrm{AA}^{\mathrm{c}}$ & 120 & 78 & 1.00 & & 1.00 & \\
\hline AG & 152 & 99 & $1.00(0.68-1.47)$ & 0.992 & $1.08(0.73-1.61)$ & 0.689 \\
\hline GG & 30 & 23 & $1.18(0.64-2.18)$ & 0.598 & $1.43(0.76-2.70)$ & 0.269 \\
\hline $\mathrm{AA}^{\mathrm{c}}$ & 120 & 78 & 1.00 & 0.869 & 1.00 & 0.506 \\
\hline AG/GG & 182 & 122 & $1.03(0.72-1.49)$ & & $1.14(0.78-1.66)$ & \\
\hline $\mathrm{AA} / \mathrm{AG}^{\mathrm{c}}$ & 272 & 177 & 1.00 & 0.576 & 1.00 & 0.302 \\
\hline GG & 30 & 23 & $1.18(0.66-2.09)$ & & $1.37(0.76-2.48)$ & \\
\hline rs2064863 & & & & 0.965 & & 0.930 \\
\hline TTc & 203 & 135 & 1.00 & & 1.00 & \\
\hline TG & 91 & 59 & $0.98(0.66-1.45)$ & 0.899 & $1.01(0.67-1.52)$ & 0.953 \\
\hline GG & 8 & 6 & $1.13(0.38-3.32)$ & 0.827 & $1.24(0.41-3.79)$ & 0.704 \\
\hline TTc & 203 & 135 & 1.00 & 0.948 & 1.00 & 0.881 \\
\hline TG/GG & 99 & 65 & $0.99(0.67-1.45)$ & & $1.03(0.69-1.53)$ & \\
\hline TT/TGc & 294 & 194 & 1.00 & 0.815 & 1.00 & 0.707 \\
\hline GG & 8 & 6 & $1.14(0.39-3.33)$ & & $1.24(0.41-3.75)$ & \\
\hline rs1047972 & & & & 0.810 & & 0.898 \\
\hline CCc & 240 & 161 & 1.00 & & 1.00 & \\
\hline CT & 59 & 36 & $0.91(0.57-1.44)$ & 0.686 & $0.93(0.58-1.50)$ & 0.769 \\
\hline TT & 3 & 3 & $1.49(0.30-7.48)$ & 0.628 & $1.33(0.26-6.82)$ & 0.733 \\
\hline $\mathrm{CC}^{\mathrm{c}}$ & 240 & 161 & 1.00 & 0.778 & 1.00 & 0.838 \\
\hline CT/TT & 62 & 39 & $0.94(0.60-1.47)$ & & $0.95(0.60-1.51)$ & \\
\hline $\mathrm{CC} / \mathrm{CT}^{\mathrm{c}}$ & 299 & 197 & 1.00 & 0.612 & 1.00 & 0.720 \\
\hline TT & 3 & 3 & $1.52(0.30-7.60)$ & & $1.35(0.26-6.89)$ & \\
\hline
\end{tabular}

Statistical analysis: logistic regression model or chi-square or Fisher's exact tests;

aCervical neoplasias consist of precancerous lesions and invasive cancer of the uterine cervix;

bThe adjusted $p$ values and adjusted odds ratios, and their $95 \%$ confident intervals were determined by logistic regression model after controlling age;

cUsed as a reference for comparison to determine the odds ratios of other genotypes. $95 \%$ CIs, $95 \%$ confidence intervals.

Table 2. Distributions of genetic polymorphism of aurora kinase A gene in women with uterine cervical invasive cancer or precancerous lesions and normal controls in Taiwan

\begin{tabular}{|c|c|c|c|c|c|c|c|c|}
\hline Genetic polymorphisms & $\begin{array}{l}\text { Normal controls } \\
(\mathrm{n}=302)\end{array}$ & $\begin{array}{l}\text { Pre-cancerous } \\
\text { lesions }(\mathrm{n}=94)\end{array}$ & $\begin{array}{l}\text { Invasive cancer } \\
(\mathrm{n}=106)\end{array}$ & $p$ values & AORs (95\% CIs) ${ }^{a}$ & Ad. $p$ values & AORs $(95 \% \text { CIs) })^{b}$ & $\begin{array}{l}\text { Ad. } p \\
\text { values }\end{array}$ \\
\hline \multicolumn{9}{|l|}{ rs2273535 } \\
\hline TTc & 138 & 42 & 54 & 0.237 & 1.00 & & 1.00 & \\
\hline TA & 134 & 40 & 48 & & $0.98(0.60-1.61)$ & 0.941 & $0.93(0.57-1.54)$ & 0.783 \\
\hline AA & 30 & 12 & 4 & & $1.32(0.62-2.80)$ & 0.476 & $0.44(0.14-1.37)$ & 0.157 \\
\hline TTc & 138 & 42 & 54 & 0.594 & 1.00 & & 1.00 & \\
\hline $\mathrm{TA} / \mathrm{AA}$ & 164 & 52 & 52 & & $1.04(0.65-1.66)$ & 0.861 & $0.85(0.52-1.38)$ & 0.512 \\
\hline $\mathrm{TT} / \mathrm{TA}^{\mathrm{c}}$ & 272 & 82 & 102 & 0.068 & 1.00 & & 1.00 & \\
\hline AA & 30 & 12 & 4 & & $1.33(0.65-2.71)$ & 0.436 & $0.46(0.15-1.38)$ & 0.165 \\
\hline rs6024836 & & & & & & & & \\
\hline
\end{tabular}




\begin{tabular}{|c|c|c|c|c|c|c|c|c|}
\hline Genetic polymorphisms & $\begin{array}{l}\text { Normal controls } \\
(\mathrm{n}=302)\end{array}$ & $\begin{array}{l}\text { Pre-cancerous } \\
\text { lesions }(\mathrm{n}=94)\end{array}$ & $\begin{array}{l}\text { Invasive cancer } \\
(\mathrm{n}=106)\end{array}$ & $p$ values & AORs (95\% CIs) ${ }^{a}$ & Ad. $p$ values & AORs $(95 \% \mathrm{CIs})^{\mathrm{b}}$ & $\begin{array}{l}\text { Ad. } p \\
\text { values }\end{array}$ \\
\hline $\mathrm{AAc}^{\mathrm{c}}$ & 120 & 32 & 46 & 0.162 & 1.00 & & 1.00 & \\
\hline AG & 152 & 46 & 53 & & $1.14(0.68-1.90)$ & 0.621 & $1.04(0.63-1.73)$ & 0.867 \\
\hline GG & 30 & 16 & 7 & & $2.01(0.98-4.14)$ & 0.059 & $0.83(0.32-2.14)$ & 0.703 \\
\hline $\mathrm{AAc}^{\mathrm{c}}$ & 120 & 32 & 46 & 0.396 & 1.00 & & 1.00 & \\
\hline AG/GG & 182 & 62 & 60 & & $1.28(0.79-2.08)$ & 0.319 & $1.01(0.62-1.66)$ & 0.956 \\
\hline $\mathrm{AA} / \mathrm{AG}^{\mathrm{c}}$ & 272 & 78 & 99 & 0.049 & 1.00 & & 1.00 & \\
\hline GG & 30 & 16 & 7 & & $1.86(0.97-3.60)$ & 0.063 & $0.81(0.33-2.00)$ & 0.651 \\
\hline \multicolumn{9}{|l|}{ rs2064863 } \\
\hline TTc & 203 & 63 & 72 & 0.896 & 1.00 & & 1.00 & \\
\hline TG & 91 & 27 & 32 & & $0.96(0.57-1.60)$ & 0.867 & $1.03(0.60-1.76)$ & 0.909 \\
\hline GG & 8 & 4 & 2 & & $1.61(0.47-5.53)$ & 0.448 & $0.78(0.15-4.12)$ & 0.772 \\
\hline TTc & 203 & 63 & 72 & 0.989 & 1.00 & & 1.00 & \\
\hline TG/GG & 99 & 31 & 34 & & $1.01(0.62-1.65)$ & 0.969 & $1.01(0.60-1.70)$ & 0.969 \\
\hline $\mathrm{TT} / \mathrm{TG}^{\mathrm{c}}$ & 294 & 90 & 104 & 0.599 & 1.00 & & 1.00 & \\
\hline GG & 8 & 4 & 2 & & $1.63(0.48-5.55)$ & 0.431 & $0.78(0.15-4.05)$ & 0.763 \\
\hline \multicolumn{9}{|l|}{ rs1047972 } \\
\hline $\mathrm{CC}^{\mathrm{c}}$ & 240 & 72 & 89 & 0.291 & 1.00 & & 1.00 & \\
\hline $\mathrm{CT}$ & 59 & 19 & 17 & & $1.07(0.60-1.92)$ & 0.811 & $0.79(0.41-1.50)$ & 0.463 \\
\hline TT & 3 & 3 & 0 & & $3.34(0.66-17.01)$ & 0.146 & u.a. & u.a. \\
\hline $\mathrm{CC}^{\mathrm{c}}$ & 240 & 72 & 89 & 0.414 & 1.00 & & 1.00 & \\
\hline $\mathrm{CT} / \mathrm{TT}$ & 62 & 22 & 17 & & $1.18(0.68-2.06)$ & 0.552 & $0.73(0.39-1.38)$ & 0.335 \\
\hline $\mathrm{CC} / \mathrm{CT}^{\mathrm{c}}$ & 299 & 91 & 106 & 0.084 & 1.00 & & 1.00 & \\
\hline $\mathrm{TT}$ & 3 & 3 & 0 & & $3.30(0.65-16.70)$ & 0.150 & u.a. & u.a. \\
\hline
\end{tabular}

aAdjusted $p$ values and adjusted odds ratios with their 95\% CIs were determined using multinomial logistic regression models after controlling age between patients with uterine cervical precancerous lesions and control women;

bAdjusted $p$ values and adjusted odds ratios with their 95\% CIs were determined using multinomial logistic regression models after controlling age between patients with uterine cervical invasive cancer and control women;

cUsed as a reference for comparison to calculate the odds ratios of other genotypes;

AORs, adjusted odds ratios; $95 \%$ CIs, $95 \%$ confidence intervals; Ad. $p$, adjusted $p$; u.a., unavailable.

Table 3. Relationships between genotypic distribution of aurora kinase A rs6024836 and clinicopathological parameters in patients with cervical invasive cancer

\begin{tabular}{|c|c|c|c|c|}
\hline \multirow[t]{2}{*}{ Parametersa } & \multicolumn{2}{|l|}{ rs6024836 } & \multirow[t]{2}{*}{$p$ value } & \multirow[t]{2}{*}{ ORs; $95 \%$ CIs } \\
\hline & $\mathrm{AA} / \mathrm{AG}^{\mathrm{b}}$ & GG & & \\
\hline Clinical stage & & & 0.136 & $1.00 ; 0.20(0.02-1.72)$ \\
\hline stage Ib & 54 & 6 & & \\
\hline$\geq$ stage II & 45 & 1 & & \\
\hline Pathologic type & & & 1.000 & 1.00; u.a. \\
\hline squamous cell carcinomab & 89 & 7 & & \\
\hline adenocarcinoma & 10 & 0 & & \\
\hline Cell grading & & & 0.093 & $1.00 ; 0.24(0.05-1.17)$ \\
\hline well (grade 1) b & 15 & 3 & & \\
\hline $\begin{array}{l}\text { moderate \& poor } \\
\text { (grades } 2 / 3 \text { ) }\end{array}$ & 84 & 4 & & \\
\hline Stromal invasion depth & & & 0.118 & $1.00 ; 0.17(0.02-1.50)$ \\
\hline$\leq 10 \mathrm{~mm}^{\mathrm{b}}$ & 50 & 6 & & \\
\hline$>10 \mathrm{~mm}$ & 48 & 1 & & \\
\hline Tumor diameter & & & 0.124 & $1.00 ; 0.18(0.02-1.59)$ \\
\hline$\leq 4 \mathrm{~cm}^{\mathrm{b}}$ & 52 & 6 & & \\
\hline$>4 \mathrm{~cm}$ & 47 & 1 & & \\
\hline Parametrium & & & 0.250 & $1.00 ; 0.26(0.03-2.21)$ \\
\hline no invasion ${ }^{\mathrm{b}}$ & 60 & 6 & & \\
\hline invasion & 39 & 1 & & \\
\hline Vagina & & & 0.237 & $1.00 ; 0.23(0.03-1.95)$ \\
\hline no invasion ${ }^{\mathrm{b}}$ & 57 & 6 & & \\
\hline invasion & 42 & 1 & & \\
\hline Pelvic lymph node & & & 0.671 & $1.00 ; 0.38(0.04-3.32)$ \\
\hline no metastasis ${ }^{b}$ & 69 & 6 & & \\
\hline metastasis & 30 & 1 & & \\
\hline
\end{tabular}

Statistical analyses: chi-square or Fisher's exact tests;

aSome clinicopathological data could not be recruited from all the patients with cervical invasive cancer due to incomplete medical charts or records;

${ }^{\mathrm{b}}$ As a reference. ORs, odds ratios; $95 \%$ CIs, $95 \%$ confidence intervals; u.a., unavailable. 
Table 4. Analysis of aurora kinase A (AURKA) genetic variants and clinicopathological characteristics in recurrence or mortality events of cervical cancer patients

\begin{tabular}{|c|c|c|c|c|c|c|c|c|}
\hline \multirow[t]{2}{*}{ Variables ${ }^{\mathrm{a}}$} & \multicolumn{4}{|c|}{ Recurrence $(\mathrm{N}=106)$} & \multicolumn{4}{|c|}{ Mortality (N=106) } \\
\hline & + & - & $p$ value & ORs (95\% CIs) & + & - & $p$ value & ORs (95\% CIs) \\
\hline AURKA rs6024836 & & & 1.000 & & & & 1.00 & \\
\hline $\mathrm{AA} / \mathrm{AG}^{\mathrm{b}}$ & 24 & 73 & & 1.00 & 77 & 25 & & 1.00 \\
\hline GG & 1 & 5 & & $0.61(0.07-5.47)$ & 1 & 0 & & u.a. \\
\hline Clinical stage & & & 0.025 & & & & $<0.001$ & \\
\hline stage $I^{b}$ & 9 & 48 & & 1.00 & 51 & 6 & & 1.00 \\
\hline$\geq$ stage II & 16 & 30 & & $2.84(1.12-7.25)$ & 27 & 19 & & $5.98(2.14-16.75)$ \\
\hline Pathologic type & & & 0.012 & & & & 0.702 & \\
\hline $\begin{array}{l}\text { squamous cell } \\
\text { carcinomab }^{\mathrm{b}}\end{array}$ & 19 & 74 & & 1.00 & 71 & 22 & & 1.00 \\
\hline adenocarcinoma & 6 & 4 & & $5.84(1.50-22.81)$ & 7 & 3 & & $1.38(0.33-5.81)$ \\
\hline Cell grading & & & 0.755 & & & & 0.755 & \\
\hline well (grade 1$)^{b}$ & 3 & 13 & & 1.00 & 13 & 3 & & 1.00 \\
\hline $\begin{array}{l}\text { moderate \& poor (grades } \\
2 / 3 \text { ) }\end{array}$ & 22 & 65 & & $1.47(0.38-5.63)$ & 65 & 22 & & $1.47(0.38-5.63)$ \\
\hline Stromal invasion depth & & & 0.001 & & & & $<0.001$ & \\
\hline$\leq 10 \mathrm{~mm}^{\mathrm{b}}$ & 6 & 47 & & 1.00 & 48 & 5 & & 1.00 \\
\hline$>10 \mathrm{~mm}$ & 19 & 30 & & $4.96(1.78-13.84)$ & 29 & 20 & & $6.62(2.24-19.55)$ \\
\hline Tumor diameter & & & 0.001 & & & & $<0.001$ & \\
\hline$\leq 4 \mathrm{~cm}^{\mathrm{b}}$ & 6 & 49 & & 1.00 & 50 & 5 & & 1.00 \\
\hline$>4 \mathrm{~cm}$ & 19 & 29 & & 5.35 (1.92-14.93) & 28 & 20 & & 7.14 (2.42-21.11) \\
\hline Parametrium & & & 0.121 & & & & 0.001 & \\
\hline no invasion ${ }^{\mathrm{b}}$ & 12 & 51 & & 1.00 & 55 & 8 & & 1.00 \\
\hline invasion & 13 & 27 & & $2.05(0.82-5.10)$ & 23 & 17 & & $5.08(1.92-13.42)$ \\
\hline Vagina & & & 0.033 & & & & 0.033 & \\
\hline no invasion ${ }^{\mathrm{b}}$ & 10 & 50 & & 1.00 & 50 & 10 & & 1.00 \\
\hline invasion & 15 & 28 & & $2.68(1.06-6.75)$ & 28 & 15 & & $2.68(1.06-6.75)$ \\
\hline Pelvic lymph node & & & 0.006 & & & & $<0.001$ & \\
\hline no metastasis & 12 & 60 & & 1.00 & 64 & 8 & & 1.00 \\
\hline metastasis & 13 & 18 & & 3.61 (1.40-9.29) & 14 & 17 & & $9.71(3.50-26.94)$ \\
\hline
\end{tabular}

Statistical analysis: Chi-square or Fisher's exact tests;

aSome clinicopathological data could not be recruited from all patients with cervical invasive cancer due to incomplete records of medical chart;

bAs a reference;

Recurrence: +, recurrence; -, no recurrence. Survival: +, survival; -, mortality. u.a.: unavailable

Table 5. Univariate analysis of the associations among aurora kinase A (AURKA) genetic polymorphism rs6024836 and various clinicopatholgical factors and the recurrence-free and overall survival of the patients with uterine cervical cancer

\begin{tabular}{|c|c|c|c|c|}
\hline \multirow[t]{2}{*}{ Variables } & \multicolumn{2}{|c|}{ Recurrence-free survival } & \multicolumn{2}{|c|}{ Overall survival } \\
\hline & $p$ value & HR \& 95\% CI ${ }^{b}$ & $p$ value & HR \& $95 \% \mathrm{CI}^{\mathrm{b}}$ \\
\hline \multicolumn{5}{|l|}{ AURKA rs6024836 } \\
\hline GG vs $\mathrm{AA} / \mathrm{AG}^{\mathrm{a}}$ & 0.656 & $0.67(0.09-4.98)$ & 0.656 & $0.68(009-5.04)$ \\
\hline \multicolumn{5}{|c|}{ Clinicopathological characteristics } \\
\hline \multicolumn{5}{|c|}{ Stage } \\
\hline$\geq$ stage II vs stage Ia & 0.026 & $2.20(0.97-4.99)$ & $<0.001$ & $3.64(1.44-9.22)$ \\
\hline \multicolumn{5}{|l|}{ Pathologic type } \\
\hline $\begin{array}{l}\text { Squamous cell carcinoma } \\
\text { adenocarcinoma }\end{array}$ & 0.006 & $2.94(1.17-7.35)$ & 0.658 & $1.53(0.45-5.16)$ \\
\hline \multicolumn{5}{|l|}{ Cell grading } \\
\hline $\begin{array}{l}\text { Well (grade } 1 \text { )a; moderate \& } \\
\text { poor (grade } 2 / 3 \text { ) }\end{array}$ & 0.577 & $1.35(0.40-4.51)$ & 0.577 & $1.49(0.45-4.98)$ \\
\hline \multicolumn{5}{|l|}{ Stromal invasion depth } \\
\hline$>10 \mathrm{~mm}$ vs $\leq 10 \mathrm{~mm}^{\mathrm{a}}$ & 0.001 & $3.43(1.37-8.58)$ & $<0.001$ & $5.46(2.03-14.67)$ \\
\hline \multicolumn{5}{|l|}{ Tumor diameter } \\
\hline$>4 \mathrm{~cm}$ vs $\leq 4 \mathrm{~cm}^{\mathrm{a}}$ & 0.001 & $3.63(1.45-9.09)$ & $<0.001$ & $5.36(2.01-14.31)$ \\
\hline \multicolumn{5}{|l|}{ Parametrium } \\
\hline $\begin{array}{l}\text { invasion vs no invasion a } \\
\text { Vagina }\end{array}$ & 0.122 & $1.71(0.78-3.74)$ & 0.001 & $3.37(1.45-7.81)$ \\
\hline invasion vs no invasiona & 0.034 & $2.09(0.94-4.66)$ & 0.034 & $2.18(0.98-4.88)$ \\
\hline Pelvic lymph node & & & & \\
\hline metastasis vs no metastasis ${ }^{a}$ & 0.006 & $2.52(1.15-5.51)$ & $<0.001$ & $6.51(2.80-15.17)$ \\
\hline
\end{tabular}

In a multivariate analysis using Cox proportional hazard model to adjust AURKA SNPs and various clincopathological factors, neither AURKA rs6024836 nor other AURKA SNPs were showed to be associated with recurrence-free survival and overall survival (Table 6). However, cervical cancer patients with adenocarcinoma (HR: 3.18, 95\% CI: $1.23-8.23 ; p=0.017$ ) and larger tumor (HR: 5.61, 95\% CI: 2.10-14.95; $p=0.001$ ) had poorer recurrence-free survival. Those with deeper stromal invasion (HR: 3.52, 95\% CI: 1.22-10.12; $p=0.020$ ) and positive pelvic lymph node metastasis (HR: 4.42, 95\% CI: 1.82-10.71; $p=0.001$ ) had poorer overall survival (Table 6).

\section{Discussion}

It was reported that AURKA was related to various cancers such as colorectal and pancreatic cancers [31, 32]. Genetic polymorphisms may display an influence on the gene expression by affecting promoter regions, exons introns, and 3'-untranslated regions [17, 21, 22]. Elevated AURKA expression might cause chromosomal instability and centrosome amplification in mammalian cells [33]. Genetic instability, probably leading to mutations of oncogenes and tumor-suppressor genes, is an 
important driving forces of malignant transformation and cancer progression [34]. AURKA overexpression may promote c-Myc oncogenic effects and cause its increased expression [35], which is associated with the development of a variety of cancers, including gynecological malignancies such as ovarian, endometrial and cervical cancers [36-38].

To date, no reports have investigated the relationships between AURKA SNPs and the development of cervical cancer. Therefore, we conducted this study, and found no different frequencies of AURKA rs2273535, rs6024836, rs2064863 and rs1047972 between patients with cervical neoplasias and normal controls. After dividing cervical neoplasias into invasive cancer and precancerous subgroups, AURKA SNPs still did not participate in the occurrence of cervical cancer. AURKA SNP rs2273535 is situated at nucleotide position 91 of exon 4 of chromosome 20q13.2 and is a non-synonymous polymorphism, encoding a phenylalanine to isoleucine substitution at amino acid position 31. Moreover, Wang et al. conducted a systemic review through the meta-analysis to reveal the involvement of AURKA rs2273535 in cancer risk and demonstrated that allele change from $\mathrm{T}$ to $\mathrm{A}$ in rs2273535 was related to an overall increased risk of cancers, especially for breast cancer [39]. rs1047972 is another nonsynonymous variant, which is also situated at exon 4, and leads to a valine to isoleucine substitution at amino acid position 57 [40]. Although AURKA SNPs may have an impact on amino acid changes, this study did not seem to show that AURKA SNPs have an influence on the development of cervical cancer. Ishikawa et al. found that an allele $\mathrm{T}$ in AURKA SNP rs2273535 and T in rs1047972 were correlated with a reduced early adverse reaction in cervical cancer patients who received pelvic radiotherapy, but this has nothing to do with the cancer itself [41]. In addition, Mesic et al. suggested that AURKA rs1047922 polymorphisms may influence the development of gastric cancer [42]. Although AURKA SNP rs758099 is located in intron area, they also found that it could have an impact on gastric cancer development.[30] Interestingly, Dai el al. used meta-analysis to indicate that AURKA SNP rs2273535 increased the risk of breast cancer especially among Asians, whereas, rs1047972 SNP reduced the risk of breast cancer in Caucasians [43].

Taking into account the relationships between AURKA SNPs and clinicopathological characteristics of cervical cancer, it showed that AURKA SNPs were not associated with these parameters. Cervical cancer patients with clinical stage $\geq$ stage II, deeper stromal invasion, larger tumor diameter, positive parametrium and vagina invasions as well as positive pelvic lymph nodes metastasis were at higher risk of mortality event. Since AURKA SNPs are not associated with these parameters, it is reasonable that AURKA SNPs are not linked to recurrence and mortality events in this study. Moreover, Chou et al. suggested that AURKA SNP rs2064863 was related to more advanced stages (stages III/IV) in patients with oral squamous cell carcinoma [24]. In contrast, rs2064863 was not concerned with tumor size, regional lymph node metastasis and distal organs metastases as well as cell differentiation. Therefore, we subdivide the stage classification of cervical cancer patients into stage I/II subgroup and III/IV subgroup to associate AURAA SNPs with cancer stages. But, it still fails to draw a significant relationship between AURKA and cancer stages (data not shown). However in hepatocellular carcinoma (HCC), Wang et al. demonstrated that patients, who carried at least one allele A in AURKA SNP rs2273535, were less likely to develop into stage III/IV disease and have large tumor. In addition, patients, who carried at least one allele G in rs2064863, exhibited a lower risk of developing large tumors. It indicated that AURKA SNPs could predict early-stage HCC [26].

Table 6. Multivariate analysis of the associations among aurora kinase A gene (AURKA) genetic polymorphism rs6024836 and various clinicopatholgical factors and the recurrence-free and overall survival of the patients with uterine cervical cancer

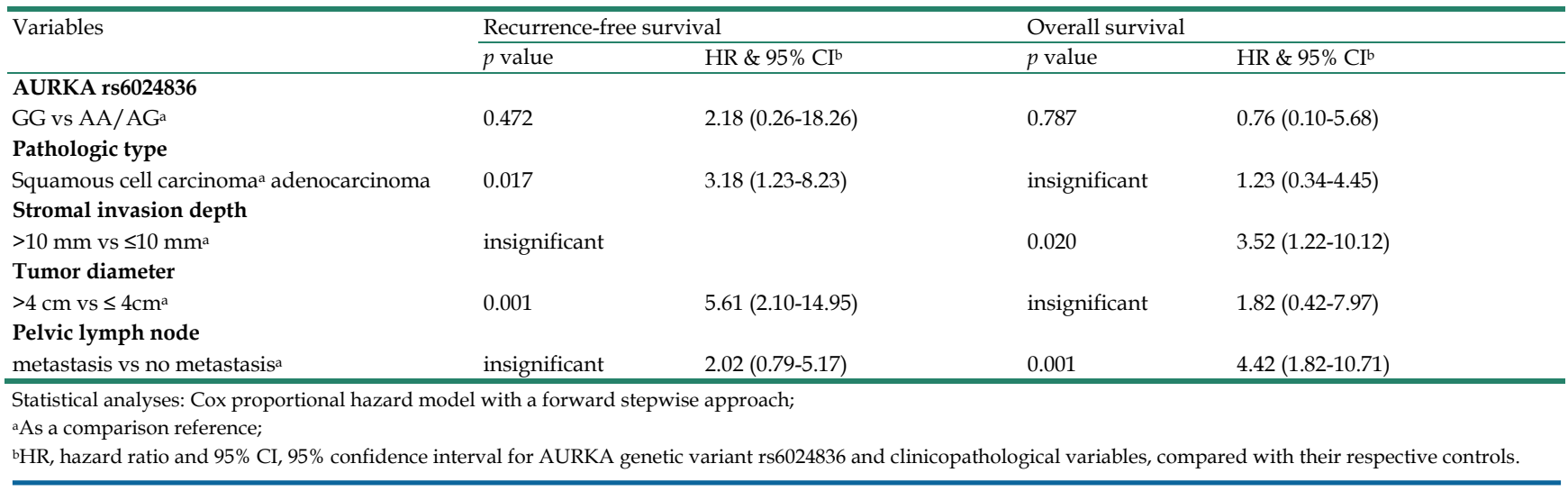


When time interval was included to correlate AURKA SNPs with prognosis, recurrence-free survival and overall survival were assessed in cervical cancer patients. In univariate Kaplan-Meier curve analysis, there were there no statistical significances between AURKA SNPs and recurrence-free survival, and overall survival. Furthermore in multivariate Cox proportional hazard model analysis, there were still no differences between AURKA SNPs and these patient prognoses. So far, no study explores the relationship of AURKA SNPs and the survival of cervical cancer patients. However in triple-negative breast cancer patients, Liao et al. found that AURKA SNPs may predict overall survival and disease-free survival [44]. Finally, we found that tumor diameter was the most obvious independent predictor of recurrence-free survival in cervical cancer patients. However, pelvic lymph node metastasis was the most obvious predictor in assessing overall survival in cervical cancer patients. It is rational because previous investigation has found that pelvic lymph node metastasis was critical in predicting patient survival of cervical cancer $[45,46]$.

In conclusion, AURKA SNPs rs2273535, rs6024836, rs2064863 and rs1047972 does not seem to be involved in the occurrence of cervical cancer in Taiwanese women. They are also not related to the clinicopathological variables of cervical cancer. These SNPs cannot predict the prognosis of cervical cancer patients. Because the studied sample was relatively small and human papillomavirus infection status lacked because of conservative attitude of Taiwanese women, it is necessary to conduct further research with a larger sample size to draw a definite conclusion.

\section{Acknowledgements}

We would like to thank the Human Biobank of Chung Shan Medical University Hospital for providing the biological specimen and related clinical data for our research. This study was supported by Chung Shan Medical University Hospital (CSH-2021E-001-Y2).

\section{Competing Interests}

The authors have declared that no competing interest exists.

\section{References}

1. Hsin MC, Hsieh $\mathrm{YH}$, Hsiao $\mathrm{YH}$, Chen PN, Wang PH, Yang SF. Carbonic Anhydrase IX Promotes Human Cervical Cancer Cell Motility by Regulating PFKFB4 Expression. Cancers 2021; 13: 1174.

2. Hsin MC, Hsieh YH, Wang PH, Ko JL, Hsin IL, Yang SF. Hispolon suppresses metastasis via autophagic degradation of cathepsin $\mathrm{S}$ in cervical cancer cells. Cell Death Dis 2017; 8: e3089.

3. Bharti AC, Shukla S, Mahata S, Hedau S, Das BC. Anti-human papillomavirus therapeutics: facts \& future. Indian J Med Res 2009; 130: 296-310.
4. Baak JP, Kruse AJ, Robboy SJ, Janssen EA, van Diermen B, Skaland I. Dynamic behavioural interpretation of cervical intraepithelial neoplasia with molecular biomarkers. J Clin Pathol 2006; 59: 1017-1028.

5. Khan MJ, Castle PE, Lorincz AT, Wacholder S, Sherman M, Scott DR, et al. The elevated 10-year risk of cervical precancer and cancer in women with human papillomavirus (HPV) type 16 or 18 and the possible utility of type-specific HPV testing in clinical practice. J Natl Cancer Inst 2005; 97: 1072-1079.

6. Kollareddy M, Dzubak P, Zheleva D, Hajduch M. Aurora kinases: structure, functions and their association with cancer. Biomed Pap Med Fac Univ Palacky Olomouc Czech Repub 2008; 152: 27-33.

7. Carmena M, Earnshaw WC. The cellular geography of aurora kinases. Nat Rev Mol Cell Biol 2003; 4: 842-854.

8. Katayama $\mathrm{H}$, Brinkley WR, Sen S. The Aurora kinases: role in cell transformation and tumorigenesis. Cancer Metastasis Rev 2003; 22: 451-464.

9. Agnese V, Bazan V, Fiorentino FP, Fanale D, Badalamenti G, Colucci G, et al. The role of Aurora-A inhibitors in cancer therapy. Ann Oncol 2007; 18 Suppl 6: vi47-52.

10. Li Z, Rana TM. A kinase inhibitor screen identifies small-molecule enhancers of reprogramming and iPS cell generation. Nat Commun 2012; 3: 1085.

11. Lee DF, Su J, Ang YS, Carvajal-Vergara X, Mulero-Navarro S, Pereira CF, et al. Regulation of embryonic and induced pluripotency by aurora kinase-p53 signaling. Cell Stem Cell 2012; 11: 179-194.

12. Sankaran S, Crone DE, Palazzo RE, Parvin JD. Aurora-A kinase regulates breast cancer associated gene 1 inhibition of centrosome-dependent microtubule nucleation. Cancer Res 2007; 67: 11186-11194.

13. Yang G, Chang B, Yang F, Guo X, Cai KQ, Xiao XS, et al. Aurora kinase A promotes ovarian tumorigenesis through dysregulation of the cell cycle and suppression of BRCA2. Clin Cancer Res 2010; 16: 3171-3181.

14. Jeng YM, Peng SY, Lin CY, Hsu HC. Overexpression and amplification of Aurora-A in hepatocellular carcinoma. Clin Cancer Res 2004; 10: 2065-2071.

15. Tanaka T, Kimura M, Matsunaga K, Fukada D, Mori H, Okano Y. Centrosomal kinase AIK1 is overexpressed in invasive ductal carcinoma of the breast. Cancer Res 1999; 59: 2041-2044.

16. Watanabe T, Imoto I, Katahira T, Hirasawa A, Ishiwata I, Emi M, et al. Differentially regulated genes as putative targets of amplifications at $20 \mathrm{q}$ in ovarian cancers. Jpn J Cancer Res 2002; 93: 1114-1122.

17. Shastry BS. SNPs: impact on gene function and phenotype. Methods Mol Biol 2009; 578: 3-22

18. Chung TT, Pan MS, Kuo CL, Wong RH, Lin CW, Chen MK, et al. Impact of RECK gene polymorphisms and environmental factors on oral cancer susceptibility and clinicopathologic characteristics in Taiwan. Carcinogenesis 2011; 32: 1063-1068.

19. Hsiao PC, Chen MK, Su SC, Ueng KC, Chen YC, Hsieh YH, et al. Hypoxia inducible factor-1alpha gene polymorphism G1790A and its interaction with tobacco and alcohol consumptions increase susceptibility to hepatocellular carcinoma. J Surg Oncol 2010; 102: 163-169.

20. Chen MK, Chiou HL, Su SC, Chung TT, Tseng HC, Tsai HT, et al. The association between hypoxia inducible factor-1alpha gene polymorphisms and increased susceptibility to oral cancer. Oral Oncol 2009; 45: e222-226.

21. Hua KT, Liu YF, Hsu CL, Cheng TY, Yang CY, Chang JS, et al. 3'UTR polymorphisms of carbonic anhydrase IX determine the miR-34a targeting efficiency and prognosis of hepatocellular carcinoma. Sci Rep 2017; 7: 4466.

22. Su CW, Chien MH, Lin CW, Chen MK, Chow JM, Chuang CY, et al. Associations of genetic variations of the endothelial nitric oxide synthase gene and environmental carcinogens with oral cancer susceptibility and development. Nitric Oxide 2018; 79: 1-7.

23. Su S, Chien M, Lin C, Chen M, Yang S. RAGE gene polymorphism and environmental factor in the risk of oral cancer. J Dent Res 2015; 94: 403-411.

24. Chou CH, Chou YE, Chuang CY, Yang SF, Lin CW. Combined effect of genetic polymorphisms of AURKA and environmental factors on oral cancer development in Taiwan. PLoS One 2017; 12: e0171583.

25. Huang CH, Chen CJ, Chen PN, Wang SS, Chou YE, Hung SC, et al. Impacts of AURKA Genetic Polymorphism on Urothelial Cell Carcinoma Development. J Cancer 2019; 10: 1370-1374.

26. Wang B, Hsu CJ, Chou CH, Lee HL, Chiang WL, Su CM, et al. Variations in the AURKA Gene: Biomarkers for the Development and Progression of Hepatocellular Carcinoma. Int J Med Sci 2018; 15: 170-175.

27. Yang PJ, Hsieh MJ, Lee CI, Yen $\mathrm{CH}$, Wang HL, Chiang WL, et al. Impact of Aurora Kinase A Polymorphism and Epithelial Growth Factor Receptor Mutations on the Clinicopathological Characteristics of Lung Adenocarcinoma. Int J Environ Res Public Health 2020; 17 :

28. Weng SL, Ng SC, Lee YC, Hsiao YH, Hsu CF, Yang SF, et al. The relationships of genetic polymorphisms of the long noncoding RNA growth arrest-specific transcript 5 with uterine cervical cancer. Int J Med Sci 2020; 17: 1187-1195.

29. Lin CW, Hsieh YS, Hsin CH, Su CW, Lin CH, Wei LH, et al. Effects of NFKB1 and NFKBIA gene polymorphisms on susceptibility to environmental factors and the clinicopathologic development of oral cancer. PLoS One 2012; 7: e35078.

30. Mesic A, Rogar M, Hudler P, Juvan R, Komel R. Association of the AURKA and AURKC gene polymorphisms with an increased risk of gastric cancer. IUBMB Life 2016; 68: 634-644.

31. Bischoff JR, Anderson L, Zhu Y, Mossie K, Ng L, Souza B, et al. A homologue of Drosophila aurora kinase is oncogenic and amplified in human colorectal cancers. EMBO J 1998; 17: 3052-3065. 
32. Li D, Zhu J, Firozi PF, Abbruzzese JL, Evans DB, Cleary K, et al. Overexpression of oncogenic STK15/BTAK/Aurora A kinase in human pancreatic cancer. Clin Cancer Res 2003; 9: 991-997.

33. Zhou H, Kuang J, Zhong L, Kuo WL, Gray JW, Sahin A, et al. Tumour amplified kinase STK15/BTAK induces centrosome amplification, aneuploidy and transformation. Nat Genet 1998; 20: 189-193.

34. Lengauer C, Kinzler KW, Vogelstein B. Genetic instabilities in human cancers. Nature 1998; 396: 643-649.

35. Lu L, Han H, Tian Y, Li W, Zhang J, Feng M, et al. Aurora kinase A mediates c-Myc's oncogenic effects in hepatocellular carcinoma. Mol Carcinog 2015; 54: 1467-1479.

36. Zheng L, Song A, Ruan Y, Chen L, Liu D, Li X, et al. Genetic polymorphisms in AURKA, BRCA1, CCNE1 and CDK2 are associated with ovarian cancer susceptibility among Chinese Han women. Cancer Epidemiol 2013; 37: 639-646.

37. Kurai M, Shiozawa T, Shih HC, Miyamoto T, Feng YZ, Kashima H, et al. Expression of Aurora kinases A and B in normal, hyperplastic, and malignant human endometrium: Aurora B as a predictor for poor prognosis in endometrial carcinoma. Hum Pathol 2005; 36: 1281-1288.

38. Sun JM, Yang LN, Xu H, Chang B, Wang HY, Yang G. Inhibition of Aurora A promotes chemosensitivity via inducing cell cycle arrest and apoptosis in cervical cancer cells. Am J Cancer Res 2015; 5: 1133-1145.

39. Wang S, Qi J, Zhu M, Wang M, Nie J. AURKA rs2273535 T>A Polymorphism Associated With Cancer Risk: A Systematic Review With Meta-Analysis. Front Oncol 2020; 10: 1040

40. Bao Z, Lu L, Liu X, Guo B, Zhai Y, Li Y, et al. Association between the functional polymorphism Ile31Phe in the AURKA gene and susceptibility of hepatocellular carcinoma in chronic hepatitis B virus carriers. Oncotarget 2017; 8: 54904-54912.

41. Ishikawa A, Suga T, Shoji Y, Kato S, Ohno T, Ishikawa H, et al. Genetic variants of NPAT-ATM and AURKA are associated with an early adverse reaction in the gastrointestinal tract of patients with cervical cancer treated with pelvic radiation therapy. Int J Radiat Oncol Biol Phys 2011; 81: 1144-1152.

42. Mesic A, Rogar M, Hudler P, Bilalovic N, Eminovic I, Komel R. Characterization and risk association of polymorphisms in Aurora kinases A, $\mathrm{B}$ and $\mathrm{C}$ with genetic susceptibility to gastric cancer development. BMC Cancer 2019; 19: 919.

43. Dai ZJ, Kang HF, Wang XI, Shao YP, Lin S, Zhao Y, et al. Association between genetic polymorphisms in AURKA (rs2273535 and rs1047972) and breast cancer risk: a meta-analysis involving 37,221 subjects. Cancer Cell Int 2014; 14: 91.

44. Liao Y, Liao Y, Li J, Li J, Fan Y, Xu B. Polymorphisms in AURKA and AURKB are associated with the survival of triple-negative breast cancer patients treated with taxane-based adjuvant chemotherapy. Cancer Manag Res 2018; 10: 3801-3808.

45. Kamura T, Tsukamoto N, Tsuruchi N, Saito T, Matsuyama T, Akazawa K, et al. Multivariate analysis of the histopathologic prognostic factors of cervical cancer in patients undergoing radical hysterectomy. Cancer 1992; 69: 181-186.

46. Choi KH, Kim JY, Lee DS, Lee YH, Lee SW, Sung S, et al. Clinical impact of boost irradiation to pelvic lymph node in uterine cervical cancer treated with definitive chemoradiotherapy. Medicine (Baltimore) 2018; 97: e0517. 